\title{
Cone-based Joint Sparse Modelling for Hyperspectral Image Classification
}

\author{
Ziyu Wang ${ }^{\mathrm{a}, \mathrm{b}}$, Rui Zhuc ${ }^{\mathrm{c}}$, Kazuhiro Fukui ${ }^{\mathrm{d}}$, Jing-Hao Xue $\mathrm{X}^{\mathrm{b}, *}$ \\ ${ }^{a}$ Department of Security and Crime Science, University College London, UK \\ ${ }^{b}$ Department of Statistical Science, University College London, UK \\ ${ }^{c}$ School of Mathematics, Statistics and Actuarial Science, University of Kent, UK \\ ${ }^{d}$ Department of Computer Science, University of Tsukuba, Japan
}

\begin{abstract}
Joint sparse model (JSM) is being extensively investigated on hyperspectral images (HSIs) and has achieved promising performance for classification. In JSM, it is assumed that neighbouring hyperspectral pixels can share sparse representations. However, the coefficients of the endmembers used to reconstruct a test HSI pixel is desirable to be non-negative for the sake of physical interpretation. Hence in this paper, we introduce the non-negativity constraint into JSM. The non-negativity constraint implies a cone-shaped space instead of the infinite sample space for pixel representation. This leads us to propose a new model called cone-based joint sparse model (C-JSM), to install the non-negativity on top of the sparse and joint modelling. To solve the C-JSM problem, we also propose a new algorithm through introducing the non-negativity constraint into the simultaneous orthogonal matching pursuit (SOMP) algorithm. The new algorithm is called non-negative simultaneous orthogonal matching pursuit (NN-SOMP). Experiments and investigations

\footnotetext{
${ }^{*}$ Corresponding author.

Email addresses: ziyu.wang.12@ucl.ac.uk (Ziyu Wang), r.zhu@kent.ac.uk (Rui
} Zhu), kfukui@cs.tsukuba.ac.jp (Kazuhiro Fukui), jinghao.xue@ucl.ac.uk (Jing-Hao Xue)
\end{abstract}


show that the proposed C-JSM can produce a more stable, sparse representation and a superior classification than other methods which only ensure the sparsity, non-negativity or spatial coherence.

Keywords: Hyperspectral image classification, joint sparse model, simultaneous orthogonal matching pursuit, cone, non-negativity.

\section{Introduction}

Sparse representation has been proven to be superiorly effective for a wide range of applications in computer vision, pattern recognition and signal processing [1, 2]. It is based on the assumption that most natural signals can be compactly represented by a linear combination of only a few basis vectors (aka atoms) from an over-complete dictionary.

Recently, sparse representation has been extensively investigated in hyperspectral imaging [3 14. A hyperspectral image (HSI) is a 3-dimensional data cube with two spatial dimensions and one spectral dimension. From the view of the spectral dimension, each HSI pixel is a vector, namely spectral signature whose elements correspond to reflectances at different wavelengths (spectral bands). Different classes of spectral signatures can have distinct reflectances at specific wavelengths and, as a result, the spectral signatures can provide discriminative information for classification. The sparse representation of an HSI pixel is accomplished by a linear combination of atoms in a spectral dictionary. The sparse model can be approximately solved by greedy algorithms such as orthogonal matching pursuit (OMP) [15] $\left(l_{0^{-}}\right.$ norm based methods) or by convex optimisation problems such as the Lasso ( $l_{1}$-norm based methods). In such sparse representation, the dictionary is usually constructed by the training spectral signatures directly from HSIs or spectral libraries. Note that, to achieve higher classification performance, 
dictionary learning has been also investigated for HSI analysis. Details of how to design and learn quality dictionaries for HSI classification can be found in [16-20], for example.

One step further, by virtue of the signal coherence in HSIs, a joint sparse model (JSM) has been successfully developed for HSI classification and has achieved promising performance [3]. The underlying assumption of JSM is that all HSI pixels in a small spatial neighbourhood can be jointly approximated by sparse linear combinations of a few common training samples, i.e. the neighbourhood shares a common sparse model. The original JSM proposed by [3] adopts a square window centred on a test pixel for joint modelling; a greedy algorithm, namely simultaneous orthogonal matching pursuit (SOMP) [21], is used to solve JSM. On top of this JSM, some extensions have been proposed to overcome the limitations of JSM [4]. To extend JSM for linearly non-separable class samples, the kernel versions of SOMP have been studied in [4, 5]. To enhance JSM with a more effective neighbourhood, the adaptive versions of JSM have been proposed in [6 9], which aim to produce shape/size adaptive local windows for JSM.

An important property of hyperspectral signals is the non-negativity, for both the signal itself and the abundance coefficients. It has been intensively considered for problems of HSI unmixing [22 36]. A large number of these reports have been focused on the non-negative matrix factorisation (NMF), a typical decomposition method for the HSI unmixing problems. NMF decomposes the sample data matrix into two low-dimensional matrices serving as endmembers and coefficients, both of which are enforced to be non-negative. The underlying assumption of the NMF-based unmixing is that mixed HSI pixels can be decomposed into a collection of endmembers and the corresponding proportions. Due to the physical characteristics, 
the endmembers, which characterise the reflected electromagnetic energy of specific materials, should be non-negative. In addition, the proportions of the underlying physical materials (endmembers) should be non-negative for physical interpretations.

From the representation point of view, a reconstructed HSI pixel by the sparse representation should be as similar as possible to the original signal, therefore is desirable to be non-negative with respect to physical interpretations. To this end, the sparse coefficients should be non-negative so long as the atoms of dictionary are non-negative. In most settings of sparse representation, the dictionary atoms are either constructed directly by the pixels in an HSI, or from a well-defined library, i.e. endmembers. Work in [37] also focuses on learning non-negative dictionaries for better representative power. This means the dictionary atoms are non-negative and hence it is reasonable to impose the non-negativity constraint on the coefficients. It is known that the non-negative coefficients estimation induces a cone-shape representation. In line with this, our recent work [38, 39] demonstrates the reasoning and appropriateness of the non-negative constraint on the coefficients, as well as the Bayesian derivations of cone-representation for HSI target detection.

However, research of sparse representation for HSI classification, particularly the JSM-based methods in [3, 6] 9], have not incorporated the nonnegativity properties of HSI. To fill in this gap, through replacing the signal representation of JSM by cone representation, in this paper we incorporate non-negativity into HSI classification and propose a new HSI classification model called cone-based joint sparse model (C-JSM).

Methodologically, inspired by the NMF for HSI unmixing, we devise the non-negativity constraint on the coefficient matrix of JSM for HSI classifi- 
cation. Since the given atoms of a dictionary are constructed directly by the HSIs or from spectral libraries, it implies that the dictionary atoms are non-negative. In this fashion, both endmembers and coefficients are non-negative, and thus the proposed C-JSM considers both sparsity and non-negativity, making the joint sparsity recovery problem more realistic in terms of interpretation. It will be illustrated to have a more sparse and stable representation than the conventional JSM.

Computationally, we propose a new algorithm called non-negative simultaneous orthogonal matching pursuit (NN-SOMP) to solve the C-JSM problem. The proposed NN-SOMP algorithm is developed on the basis of the SOMP algorithm with an additional non-negative constraint on the coefficients, which will be illustrated easy to implement in this paper.

In short, the main contribution of this paper can be summarised as follows: 1) we incorporate the non-negativity constraints into JSM to consider more realistic physical characteristics of the spectral signals and propose a new HSI classification model called C-JSM; 2) we also propose a new NNSOMP algorithm to solve the optimisation problem of C-JSM; and 3) C-JSM produces a stable sparse representation as well as a superior classification performance.

The rest of the paper is organised as follows. Section 2 reviews the sparse model (SM) and the joint sparse model (JSM) for the HSI classification. Section 3 introduces the cone-based model and the cone-based sparse model. In section 4, the proposed C-JSM, as well as the proposed algorithm NNSOMP to solve the C-JSM problem, are detailed. Experimental studies in section 6 demonstrates the superior classification performance of C-JSM over the compared methods on two real hyperspectral datasets. Finally this work is discussed and concluded in section 7 . 


\section{Joint sparse models for HSI classification}

\subsection{Sparse model}

Given an unknown $B$-dimensional signal $\mathbf{x} \in \mathbb{R}^{B}$ and a dictionary constructed by $N$ training samples (also termed atoms) $\mathbf{D}=\left[\mathbf{d}_{1}, \ldots, \mathbf{d}_{N}\right] \in$ $\mathbb{R}^{B \times N}$ with $B<N$, the sparse model is formulated as finding a sparse coefficient vector $\boldsymbol{\alpha} \in \mathbb{R}^{N}$ that

$$
\mathrm{x} \approx \mathbf{D} \alpha
$$

The sparse coefficient vector $\boldsymbol{\alpha}$ implies that the signal is approximated by the linear combination of only a small number of (e.g. at most $L$ ) atoms in $\mathbf{D}$. It can be estimated by solving the following optimisation problem:

$$
\hat{\boldsymbol{\alpha}}=\underset{\boldsymbol{\alpha}}{\operatorname{argmin}}\|\mathbf{x}-\mathbf{D} \boldsymbol{\alpha}\|_{2} \text {, s.t. }\|\boldsymbol{\alpha}\|_{0} \leq L .
$$

In $(2),\|\boldsymbol{\alpha}\|_{0}$ denotes a $l_{0}$-pseudo-norm of $\boldsymbol{\alpha}$, which indicates the number of non-zero elements in $\boldsymbol{\alpha}$; and $L(L \ll N)$ denotes the sparsity level of the model. The problem in (2) is NP-hard, but it can be approximately solved by greedy algorithms such as orthogonal matching pursuit (OMP) [15], or be relaxed by replacing the $l_{0}$-pseudo-norm with the $l_{1}$-norm such as the Lasso. It is worth noting that the dictionary $\mathbf{D}$ have unit $l_{2}$-norm columns.

\subsection{Joint Sparse model (JSM)}

In HSIs, local smoothness and sparsity are often assumed, because neighbouring pixels in a small area often consist of similar materials and the classes of these materials are few. The joint sparse model (JSM) is proposed under these assumptions, with all neighbouring pixels around a central pixel sharing a common sparsity pattern. 
Instead of sparsely representing a single signal vector $\mathbf{x} \in \mathbb{R}^{B}$, the JSM is designed to model for a matrix $\mathbf{X} \in \mathbb{R}^{B \times T}$ of $T$ signals simultaneously, and assumes that the signals share a common sparse pattern. In the classification of HSI, the matrix $\mathbf{X}$ denotes a small window centring on a test pixel $\mathbf{x}_{c}$ and consisting of $T$ HSI pixels, with each pixel $\mathbf{x}_{t}$ for $t=1, \ldots, T$ represented by a $B$-dimensional vector for $B$ spectral bands. The $T$ pixels in the window are jointly approximated by sparse linear combinations of atoms from a given over-complete dictionary $\mathbf{D}$ :

$$
\mathbf{X}=\left[\mathbf{x}_{1}, \ldots, \mathbf{x}_{T}\right] \approx \mathbf{D}\left[\boldsymbol{\alpha}_{1}, \ldots, \boldsymbol{\alpha}_{T}\right]=\mathbf{D A}
$$

where $\mathbf{A} \in \mathbb{R}^{N \times T}$ is the matrix of unknown coefficients $\left[\boldsymbol{\alpha}_{1}, \ldots, \boldsymbol{\alpha}_{T}\right]$. It is assumed that the coefficient vectors share the same sparsity pattern, i.e. A only contains $L(L \ll N)$ non-zero rows.

The estimation of $\mathbf{A}$ can be achieved by solving a joint sparse recovery problem:

$$
\hat{\mathbf{A}}=\underset{\mathbf{A}}{\operatorname{argmin}}\|\mathbf{X}-\mathbf{D A}\|_{F}^{2} \text {, s.t. }\|\mathbf{A}\|_{\text {row }, 0} \leq L,
$$

where $\|\cdot\|_{F}$ denotes the Frobenius norm; and $\|\mathbf{A}\|_{\text {row }, 0}$ denotes the row-wise $l_{0}$-pseudo-norm, which is the number of non-zero rows of $\mathbf{A}$. An illustration of the JSM equation is shown in Figure. 1. As with (2), problem (4) is NP-hard and it can be approximately solved by greedy algorithms such as simultaneous orthogonal matching pursuit (SOMP) algorithm [21]. The dictionary $\mathbf{D}$ is also column-wise normalised to have unit $l_{2}$-norm. 


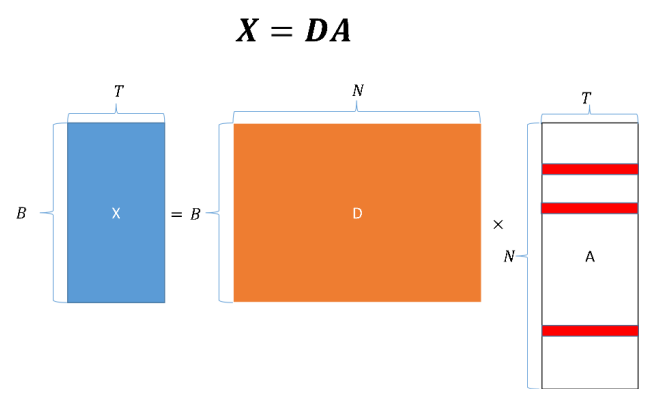

Figure 1: An illustration of JSM, where $\mathbf{X}$ is a $B \times T$ matrix denoting a small window consisting of $T$ HSI pixels, $\mathbf{D}$ is a $B \times N$ matrix representing an over-complete dictionary with $N$ atoms; and $\mathbf{A}$ is an $N \times T$ coefficient matrix with only $L$ non-zero rows. The red lines in $\mathbf{A}$ indicate the non-zero rows and the blank areas indicate zero rows of $\mathbf{A}$.

\subsection{Classification rules of SM and JSM}

For the SM, the class of $\mathbf{x}$ is determined by applying the obtained sparse coefficient vector $\hat{\boldsymbol{\alpha}}$ from (2). We define the class-wise residuals as

$$
r^{m}(\mathbf{x})=\left\|\mathbf{x}-\mathbf{D}^{m} \hat{\boldsymbol{\alpha}}^{m}\right\|_{2}^{2}, m=1, \ldots, M,
$$

where $M$ is the total number of classes, $\hat{\boldsymbol{\alpha}}^{m}$ contains the $N_{m}$ elements in $\hat{\boldsymbol{\alpha}}$ that are associated with sub-dictionary $\mathbf{D}^{m}$ of the $m$ th class, with $N=$ $\sum_{m=1}^{M} N_{m}$. The label of the test pixel $\mathbf{x}$ is determined by its minimal residual over all $M$ classes:

$$
\operatorname{Class}(\mathbf{x})=\underset{m=1, \ldots, M}{\operatorname{argmin}} r^{m}(\mathbf{x}) .
$$

For the JSM, once the sparse coefficient matrix $\hat{\mathbf{A}}$ is obtained from (4), we calculate the class-wise residual of the matrix $\mathbf{X}$ from its class-wise approximation similar to (5):

$$
r^{m}(\mathbf{X})=\left\|\mathbf{X}-\mathbf{D}^{m} \hat{\mathbf{A}}^{m}\right\|_{F}^{2}, m=1, \ldots, M
$$


In (7), there are $N_{m}$ rows in $\hat{\mathbf{A}}$ corresponding to a sub-dictionary $\mathbf{D}^{m}$ and $N=\sum_{m=1}^{M} N_{m}$. Different from the SM, the label of the central test pixel $\mathbf{x}_{c}$ in window $\mathbf{X}$ is jointly determined by the minimal residual of $\mathbf{X}$ over all $M$ classes, i.e.

$$
\operatorname{Class}\left(\mathbf{x}_{c}\right)=\underset{m=1, \ldots, M}{\operatorname{argmin}} r^{m}(\mathbf{X}) .
$$

\section{Cone-based sparse model}

\subsection{Cone-based model}

A cone model $(\mathrm{CM})$ to represent vectors $\mathbf{x}$ is defined as

$$
\mathbf{C}:\left\{\mathbf{x} \mid \mathbf{x}=\sum_{i=1}^{N} \alpha_{i} \mathbf{d}_{i}=\mathbf{D} \boldsymbol{\alpha}, \alpha_{i} \geq 0\right\}
$$

where $\alpha_{i}$ is the non-negative coefficient of atom $\mathbf{d}_{i}$, and $\boldsymbol{\alpha}$ is an $N$-dimensional vector of non-negative coefficients.

The non-negative coefficient vector $\boldsymbol{\alpha}$ is estimated by solving the following optimisation problem:

$$
\hat{\boldsymbol{\alpha}}=\underset{\boldsymbol{\alpha}}{\operatorname{argmin}}\|\mathbf{x}-\mathbf{D} \boldsymbol{\alpha}\|_{2}^{2}, \text { s.t. } \boldsymbol{\alpha} \geq \mathbf{0},
$$

where $\boldsymbol{\alpha} \geq \mathbf{0}$ denotes that every single element of the vector $\boldsymbol{\alpha}$ should be non-negative. Problem (10) can be solved by the active-set methods, such as the typical non-negative least square method (NNLS) [40] (MATLAB function lsqnonneg) and its extension fast-NNLS (fnnls) [41]. In this paper, we use the CM (10) as a baseline method for HSI classification. Specifically, (10) is used for the representation of a single test HSI pixel. The label of a test pixel is determined by (6), as with the rule used by the SM (2). 


\subsection{Cone-based sparse model}

For the $l_{0}$-pseudo-norm optimisation problem, the non-negative orthogonal matching pursuit (NN-OMP) algorithm has been investigated in [42], which introduces the non-negativity constraint into the conventional OMP algorithm. Technical details of the algorithm vary, depending on different criteria such as fast implementation [43].

In [42, 43], a desired coefficient vector $\boldsymbol{\alpha}$ is estimated by solving the following optimisation problem:

$$
\hat{\boldsymbol{\alpha}}=\underset{\boldsymbol{\alpha}}{\operatorname{argmin}}\|\mathbf{x}-\mathbf{D} \boldsymbol{\alpha}\|_{2}^{2}+\|\boldsymbol{\alpha}\|_{0}, \text { s.t. } \boldsymbol{\alpha} \geq \mathbf{0},
$$

which is forced to be sparse and non-negative.

In this paper, we term model (11) the cone-based sparse model (shortened as CSM). To our knowledge, CSM is first introduced and studied in this paper for HSI classification. To align with the rule of SM (2), the classification of an HSI based on CSM (11) is also determined by (6).

\section{Cone-based joint sparse model (C-JSM) for HSI classification}

We notice that, on the one hand, SM (2), CM (10) and CSM (11) are all constructed for a single test HSI pixel and do not take the spatial coherence [44] into consideration; while on the other hand, JSM accounts for the neighbouring spatial information, but the coefficients estimated by JSM are only assumed to be sparse, not necessarily non-negative. As with the underlying assumptions made for HSI unmixing, an HSI pixel can be decomposed into a collection of endmembers with non-negative proportions. The endmembers are spectral signatures which characterise the reflect electromagnetic energy of specific materials and hence are non-negative. In 
the case of HSI classification, the dictionary atoms are usually constructed directly from the HSI or from the spectral libraries, so the atoms can be assumed acting as endmembers, which inspires us to devise a cone-based representation for the joint models for a more realistic interpretation.

In the same notation as aforementioned, the cone-based representation of a test window $\mathbf{X} \in \mathbb{R}^{B \times T}$ can be formulated as follows:

$$
\mathbf{X} \approx \mathbf{D A} \text {, s.t. } \mathbf{A} \geq \mathbf{0}
$$

where $\mathbf{A}$ is a non-negative coefficient matrix and $\mathbf{A} \geq \mathbf{0}$ denotes that every element of $\mathbf{A}$ should be non-negative. To estimate $\mathbf{A}$, problem 12 can be reformulated as

$$
\hat{\mathbf{A}}=\underset{\mathbf{A}}{\operatorname{argmin}}\|\mathbf{X}-\mathbf{D A}\|_{F}^{2} \text {, s.t. } \mathbf{A} \geq \mathbf{0} \text {. }
$$

In this paper, we term model (12) the joint cone model (shortened as JCM). We also utilise it as a baseline method.

The optimisation problem (13) can be solved by two algorithms. Firstly, the reconstruction of each column vector $\mathbf{x}_{t}$ for $t=1, \ldots, T$ can be solved independently by the conventional NNLS [40] or fast-NNLS [41]. Secondly, it can be solved by an algorithm called fast combination NNLS (FC-NNLS) [45], which is proposed to solve the large-scaled non-negativity-constrained least square problems. It solves a set of linear reconstruction for $\mathbf{X}$ in a parallel fashion instead of solving a set of single $\mathbf{x}$ in a serial fashion. Specifically, it rearranges the calculations in the standard active-set NNLS on the basis of combinational reasoning and reduces the computation burden for NNLS problems when there are a large number of observations, i.e. a large win- 
dow size $T$ in our case. The estimated coefficient matrices $\hat{\mathbf{X}}_{\text {s obtained by }}$ these two methods are the same. So we can regard FC-NNLS as a fast implementation of NNLS for solving the JCM problem (13).

Incorporating JCM (13) into the JSM for HSI classification (4), we propose a new method as

$$
\begin{gathered}
\qquad \hat{\mathbf{A}}=\underset{\mathbf{A}}{\operatorname{argmin}}\|\mathbf{X}-\mathbf{D A}\|_{F}^{2}, \\
\text { s.t. }\|\mathbf{A}\|_{\text {row }, 0} \leq L \text { and } \mathbf{A} \geq \mathbf{0} .
\end{gathered}
$$

We call this new model (14) the cone-based joint sparse model (shortened as C-JSM). In short, the proposed C-JSM incorporates the non-negative constraints into the sparse representation of a test window $\mathbf{X}$ by joint modelling. The coefficient matrix $\mathbf{A}$ of the test window $\mathbf{X}$ is not only sparse, but also forced to be non-negative. On top of these two desirable properties, the spatial coherence of HSI is also reflected in that the coefficient vector of the central test pixel $\mathbf{x}_{c}$ is jointly determined by those HSI pixels in its local neighbourhood with the same non-negative and sparse constraints. As a result, HSI pixels in the local window $\mathbf{X}$ share the same basis vectors of a cone, and the sparsity of the coefficients are determined only in the region of the cone.

Same as JSM, the two cone-based sparse models, JCM and C-JSM, are also joint models, hence we adopt the classification rule 8 for them. To solve the C-JSM problem (14), we propose a new algorithm and detail it in the following section 5 . 


\section{Algorithm of NN-SOMP for solving C-JSM}

We propose a new algorithm called non-negative simultaneous orthogonal matching pursuit (NN-SOMP), to solve the C-JSM problem. It combines the NNLS-based methods and the SOMP algorithm together to produce a non-negative and sparse estimation of the coefficient matrix $\hat{\mathbf{A}}$ in 14 . Before introducing the proposed NN-SOMP, we first present the non-negative OMP to get an insight of the paradigm.

\subsection{Algorithm of $\mathrm{NN}-\mathrm{OMP}$}

The traditional $\mathrm{SM}$ in (2) with the $l_{0}$-pseudo-norm constraint on the coefficient vector is approximately solved by greedy algorithms, of which one of the most popular algorithms is called orthogonal matching pursuit (OMP) [15. We assume that the columns (atoms) of the dictionary $\mathbf{D}$ are normalised so that $\left\|\mathbf{d}_{i}\right\|_{2}=1$ for $i=1, \ldots, N$. At the beginning of the algorithm, a residual vector $\mathbf{r}_{0}$ is initialised to be the test HSI pixel $\mathbf{x}$. The OMP iteratively selects at each step the column of $\mathbf{D}$, i.e. the atom $\mathbf{d}_{i}$, which has not been selected but is most correlated with the residuals $\mathbf{r}_{j-1}$, where $j$ is the current iteration number. The maximal correlation is calculated as $\left|\mathbf{d}_{i}^{T} \mathbf{r}_{j-1}\right|$, which is the absolute value of the projection of residual vector $\mathbf{r}_{j-1}$ onto the the atom $\mathbf{d}_{i}$. The selected atom $\mathbf{d}_{i}$ is then added into the set of selected atoms. The algorithm updates the residual vector by projecting the observed vector $\mathbf{x}$ onto the linear subspace spanned by the atoms that have already been selected, and then iterates. The termination of the OMP algorithm is either conducted by setting the iteration number, i.e. the sparsity level $L$, or by setting a threshold $\tau$ of the residual.

Based on the OMP algorithm, the non-negative OMP (NN-OMP) is proposed by incorporating the non-negativity constraint on the coefficients 
into the iterations. The main difference between OMP and NN-OMP is the updating criteria of residual vector $\mathbf{r}_{j}$. In OMP, the residual vector is

updated by $\mathbf{r}_{j}=\mathbf{x}-\mathbf{D}_{\Lambda_{j}} \hat{\boldsymbol{\beta}}_{j}$, where the coefficient vector $\boldsymbol{\beta}_{j}$ is obtained by least squares (LS) and has a closed-form solution. However in NN-OMP, to guarantee non-negative coefficients, the coefficient vector $\boldsymbol{\beta}_{j}$ at iteration $j$ should be solved by NNLS-based methods instead of the LS method, which is described in (16). Hence there is no closed-form solution for $\boldsymbol{\beta}_{j}$. The algorithm of NN-OMP used in this paper is summarised in Algorithm 1 .

Other versions of NN-OMP can be found in [42,43]. We note that there is a slight difference between Algorithm 1 and the algorithms proposed in [42, 43]: we use the absolute value $\left|\mathbf{d}_{i}^{T} \mathbf{r}_{j-1}\right|$ instead of the maximal positive value $\max \left(\mathbf{d}_{i}^{T} \mathbf{r}_{j-1}\right)>0$ used in [42, 43]. Although these two approaches may select different atoms from iteration 2 (for iteration $1, \mathbf{r}_{0}$ and $\mathbf{d}_{i}$ both are positive so the produced results are same), the size of residuals $\left\|\mathbf{r}_{j}\right\|_{2}$ can be reduced iteratively by both, which reflects the core idea of matching pursuit algorithms. It may not be easy to claim which approach is more appropriate. To align with the original framework of OMP and for a clearer comparison, we only change the updating of the coefficients by (16) and adopt Algorithm 1 as a representative of NN-OMP algorithm in the following discussion.

\subsection{Algorithm of $N N-S O M P$}

Following the derivation of NN-OMP from OMP, we propose a new algorithm called NN-SOMP, which combines the SOMP algorithm [21] and the NNLS-based methods together to solve the problem of C-JSM (14).

The SOMP algorithm [21] is a generalised OMP algorithm. It aims to find a simultaneous approximation of several input signals, i.e. several 
Algorithm 1 The NN-OMP algorithm to solve CSM (11).

Input: $\quad$ Dictionary $\mathbf{D}=\left[\mathbf{d}_{1}, \ldots, \mathbf{d}_{N}\right] \in \mathbb{R}^{B \times N}$ with $\left\|\mathbf{d}_{i}\right\|_{2}^{2}=1$ for $i=$ $1, \ldots, N$.

- A test pixel $\mathbf{x} \in \mathbb{R}^{B}$.

- Sparsity level $L$ or threshold $\tau$.

Output: A non-negative and sparse coefficient vector $\hat{\boldsymbol{\alpha}}$.

\section{Initialisation:}

- The residual vector $\mathbf{r}_{0}=\mathbf{x}$.

- Sparse index set $\Lambda_{0}=\varnothing$.

- Iteration counter $j=1$.

while $j \leqslant L$ or $\left\|\mathbf{r}_{j-1}\right\|_{2}^{2}<\tau$ do

(1) Find an index $\lambda_{j}$ that solves the easy optimisation problem:

$$
\lambda_{j}=\underset{i=1, \ldots, N}{\operatorname{argmax}}\left|\mathbf{d}_{i}^{T} \mathbf{r}_{j-1}\right| .
$$

(2) Update the index set $\Lambda_{j}=\Lambda_{j-1} \cup\left\{\lambda_{j}\right\}$.

(3) Determine non-negative coefficient vector $\boldsymbol{\beta}_{j}$ by the NNLS algorithm in the cone $C$ whose basis vectors are the atoms of $\mathbf{D}$ indexed in $\Lambda_{j}$ :

$$
\hat{\boldsymbol{\beta}}_{j}=\underset{\boldsymbol{\beta}_{j}}{\operatorname{argmin}}\left\|\mathbf{x}-\mathbf{D}_{\Lambda_{j}} \boldsymbol{\beta}_{j}\right\|_{2}^{2} \text {, s.t. } \boldsymbol{\beta}_{j} \geq \mathbf{0},
$$

where $\mathbf{D}_{\Lambda_{j}} \in \mathbb{R}^{B \times j}$ consists of the $j$ atoms in $\mathbf{D}$ indexed in $\Lambda_{j}$.

(4) Determine the new residual:

$$
\mathbf{r}_{j}=\mathbf{x}-\mathbf{D}_{\Lambda_{j}} \hat{\boldsymbol{\beta}}_{j}
$$

(5) $j \leftarrow j+1$.

end while

Compute the non-negative and sparse coefficient vector $\hat{\boldsymbol{\alpha}}$ whose non-zero elements are indexed by $\Lambda$ and the corresponding $L$ elements of vector $\hat{\boldsymbol{\beta}}_{L}$. 
columns of matrix $\mathbf{X}$, by using different linear combinations of the same atoms of the dictionary. The algorithm balances the error in approximation against the total number of atoms that participate. Specifically, the atoms supporting the sparse solution are sequentially selected from the dictionary. At each iteration, the atom that simultaneously yields the best yet simple approximation to all of the residual vectors is selected. Particularly, at the $j$ th iteration, we calculate an $N \times T$ correlation matrix $C o r r=\mathbf{D}^{T} \mathbf{R}_{j-1}$, where $\mathbf{R}_{j-1}$ is a residual matrix between the test window $\mathbf{X} \in \mathbb{R}^{B \times T}$ and its approximation from the last iteration. The $(i, t)$ th entry in Corr is the correlation between the $i$ th dictionary atom $\mathbf{d}_{i}$ and the residual vector for $\mathbf{x}_{t}$, where $t=1, \ldots, T$ at the current iteration $j$. In the algorithm, the $l_{p}$-norm, where $p \geq 1$, for each of the $N$ rows of $\operatorname{Corr}$ is computed. The row index corresponding to the largest $l_{p}$-norm is then added into the sparse index set of selected atoms. As mentioned in [3], different values of $p$ have been adopted in literatures, such as $p=1$ is in [21], $p=2$ in [46] and $p=\infty$ in [47]. In this paper we use $p=\infty$ to align with [47]. Similarly to OMP, the termination of the SOMP algorithm is either conducted by setting the iteration number, i.e. the sparsity level $L$, or by setting a threshold $\tau$ of the size of the residual. Details of the SOMP algorithm can also be found in [3].

The proposed NN-SOMP algorithm is devised on the basis of the SOMP algorithm; it incorporates non-negative constraints in the simultaneous approximation of a test window $\mathbf{X}$ and is summarised in Algorithm 2 . We replace the LS-based estimates of the coefficient matrix of SOMP by the NNLS-based estimates (19). We can see that (19) in our proposed algorithm is in fact a standard JCM problem as in 13.

As aforementioned, the optimisation problem (19) can be solved by two strategies both based on the NNLS methods. For a simple implementa- 
tion, each column of $\mathbf{X}$ can be treated independently. Specifically, problem (19) in the Algorithm 2 is broken into $T$ individual NNLS problems formulated by (10). These $T$ problems can be solved by conventional NNLS algorithm [40] or fast-NNLS algorithm [41]. Then the coefficient matrix $\hat{\mathbf{P}}_{j}$ is obtained by concatenating the estimated coefficient vectors column by column. In this fashion, we need to use an inner FOR loop to compute step (3) of the NN-SOMP algorithm (Algorithm 2). The optimisation problem 19 can also be solved by the FC-NNLS algorithm [45], which is a generalised NNLS algorithm. It aims to solve the non-negative least squares with multiple input vectors. FC-NNLS rearranges the selection of the support set, and reduces substantially the computational burden required for the NNLS problems which have large numbers of observation vectors.

The conventional NNLS algorithm utilises the active/passive set method to solve an inequality-constrained least squares problem as a sequence of equality-constrained problems, also termed "column-serial" 45]. FC-NNLS is also based on this NNLS scheme. In general, the overall NNLS in the FC-NNLS is responsible for defining the sequence, but sequentially solving the problem tends to be computationally inefficient as it can result in redundant calculations. To this end, FC-NNLS solves the problem in a "column-parallel" fashion. Specifically, the algorithm firstly groups problems that share a common passive set and solve them together, and then recognises that the passive sets vary from iteration to iteration. Each NNLS iteration for all columns are performed in parallel rather than performing all iterations for each column in series. Note that columns will require different numbers of iterations to achieve optimality. The conventional NNLS and the FC-NNLS produce the same estimation results; for a faster computation, we adopt the FC-NNLS algorithm to solve (19). 
Algorithm 2 The NN-SOMP algorithm to solve C-JSM (14).

Input: $\quad$ Dictionary $\mathbf{D}=\left[\mathbf{d}_{1}, \ldots, \mathbf{d}_{N}\right] \in \mathbb{R}^{B \times N}$ with $\left\|\mathbf{d}_{i}\right\|_{2}^{2}=1$ for $i=$ $1, \ldots, N$.

- A test window $\mathbf{X}=\left[\mathbf{x}_{1}, \ldots, \mathbf{x}_{T}\right] \in \mathbb{R}^{B \times T}$.

- Sparsity level $L$.

Output: A non-negative and sparse coefficient matrix $\hat{\mathbf{A}}$.

\section{Initialisation:}

- The residual matrix $\mathbf{R}_{0}=\mathbf{X}$.

- Sparse index set $\Lambda_{0}=\varnothing$.

- Iteration counter $j=1$.

while $j \leqslant L$ or $\left\|\mathbf{R}_{j-1}\right\|_{F}^{2}<\tau$ do

(1) Find an index $\lambda_{j}$ that solves the following easy optimisation problem:

$$
\lambda_{j}=\underset{i=1, \ldots, N}{\operatorname{argmax}}\left\|\mathbf{R}_{j-1}^{T} \mathbf{d}_{i}\right\|_{p}, p \geq 1 .
$$

(2) Update the index set $\Lambda_{j}=\Lambda_{j-1} \cup\left\{\lambda_{j}\right\}$.

(3) Determine non-negative coefficient matrix $\mathbf{P}_{j}$ by the NNLS-based algorithm in the cone $C$ whose basis vectors are the atoms of $\mathbf{D}$ indexed in $\Lambda_{j}$ :

$$
\hat{\mathbf{P}}_{j}=\underset{\mathbf{P}_{j}}{\operatorname{argmin}}\left\|\mathbf{X}-\mathbf{D}_{\Lambda_{j}} \mathbf{P}_{j}\right\|_{F}^{2}, \text { s.t. } \mathbf{P}_{j} \geq \mathbf{0}
$$

where $\mathbf{D}_{\Lambda_{j}} \in \mathbb{R}^{B \times j}$ consists of number of $j$ atoms in $\mathbf{D}$ indexed in $\Lambda_{j}$. Optimisation problem $(19)$ can either determined in a serial fashion that each column of $\mathbf{X}$ is treated independently and can be approximated by NNLS [40] or fast-NNLS [41]; or in a parallel fashion by FC-NNLS [45]. The two approaches produce the same result.

(4) Determine the new residual matrix:

$$
\mathbf{R}_{j}=\mathbf{X}-\mathbf{D}_{\Lambda_{j}} \hat{\mathbf{P}}_{j}
$$

(5) $j \leftarrow j+1$.

end while

Compute the non-negative and sparse coefficient matrix $\hat{\mathbf{A}}$ whose nonzeros rows are indexed by $\Lambda$ and the corresponding $L$ rows of matrix $\hat{\mathbf{P}}_{L}$. 


\section{Experiments}

In this section, we investigate the performance of the proposed C-JSM method on HSI classification. The experiments are carried out on two wellknown real HSI datasets: the AVIRIS Indian Pines dataset and the ROSIS University of Pavia dataset, both of which can be downloaded from [48].

\subsection{Methods compared}

We evaluate the proposed C-JSM (14) and compare it with five baseline methods: the sparse model (SM) (2), the joint sparse model (JSM) (4), the cone model (CM) (10), the cone-based sparse model (CSM) (11) and the joint cone model (JCM) (13). Corresponding algorithms used to learn these models are listed in Table 1 the proposed NN-SOMP (Algorithm 2), OMP [15], SOMP [21], NNLS [40], NN-OMP (Algorithm[1) and FC-NNLS [45], respectively.

Table 1: Compared methods and their corresponding algorithms: CM - cone model; SM - sparse model; CSM - cone-based sparse model; JCM - joint cone model; JSM - joint sparse model; C-JSM - cone-based joint sparse model.

\begin{tabular}{l|r|r|r|r|r|r}
\hline Meth. & CM & SM & CSM & JCM & JSM & C-JSM \\
\hline Alg. & NNLS & OMP & NN-OMP & FC-NNLS & SOMP & NN-SOMP \\
\hline
\end{tabular}

From the point of view of models, these six methods can be grouped into two types of models: single models (CM, SM, and CSM) and joint models (JCM, JSM and C-JSM). The single models label a test HSI pixel by considering only the test pixel, i.e. a vector $\mathrm{x}$ in (2), (10) and (11), whereas the joint models label a central test HSI pixel $\mathbf{x}_{c}$ by considering a local window around it, i.e. a matrix $\mathbf{X}$ in (4), (13) and (14). The labelling by the single models is determined by (6), whereas the labelling by the joint models is determined by (8). The compared methods can also be grouped 
according to their constraints on non-negativity and sparsity: CM and JCM are only with the non-negativity constraint; SM and JSM are only with the sparsity constraint; and CSM and C-JSM both consider the non-negativity and sparsity simultaneously. Details of the relationships among the methods, algorithms, models and constraints are presented in the confusion matrices in Table 2 and Table 3 ,

Table 2: Compared methods and their groups.

\begin{tabular}{r|r|r|r}
\hline & Non-negative & Sparse & Non-negative + Sparse \\
\hline Single model & CM & SM & CSM \\
\hline Joint model & JCM & JSM & C-JSM \\
\hline
\end{tabular}

Table 3: Compared algorithms and their groups.

\begin{tabular}{r|r|r|r}
\hline & Non-negative & Sparse & Non-negative + Sparse \\
\hline Single model & NNLS & OMP & NN-OMP \\
\hline Joint model & FC-NNLS & SOMP & NN-SOMP \\
\hline
\end{tabular}

\subsection{Performance measures}

We evaluate the performances of the compared methods by using three standard measures for HSI classification: the overall accuracy $(O A)$, the average accuracy $(A A)$ and kappa coefficient $\kappa$ [49], which are widely used by the remote sensing community.

The $O A, A A$ and $\kappa$ are defined as follows:

$$
O A=\frac{N_{\text {corr }}}{N_{\text {test }}}, A A=\frac{1}{M} \sum_{m=1}^{M} \frac{N_{m}^{\text {corr }}}{N_{m}^{\text {class }}} \text { and } \kappa=\frac{O A-p_{e}}{1-p_{e}}
$$

In (21), the overall accuracy $(O A)$ is defined as the ratio of the number of the correctly-classified test pixels $N_{\text {corr }}$ over the total number of test pixels $N_{\text {test }}$. The average accuracy $(A A)$ is defined as the average value of $M$ 
accuracies of the $M$ individual classes, where $N_{m}^{\text {class }}$ is the total number of test pixels of class $m$, and $N_{m}^{c o r r}$ is the number of the correctly-classified test pixels of class $m$. The $\kappa$ coefficients measures the percentage of classified test pixels corrected by the number of agreements that would be expected purely by change [49]. In 21], we have $p_{e}=\sum_{m=1}^{M}\left(F_{m} \times F_{m}^{t}\right)$, where $F_{m}$ is the ratio of data assigned to class $m$ by the classifier and $F_{m}^{t}$ is the ratio of data that belong to class $m$.

\subsection{Parameter settings}

Among the compared methods, in single models, only one unknown parameter needs to be determined, i.e. the sparsity level $L$; in joint models, two unknown parameters are involved, the sparsity level $L$ and the window size $T$, except for FC-NNLS in which only the window size $T$ is involved. The values of the parameters for all methods are determined via the leaveone-out cross validation (LOOCV) in the training phase.

\subsection{Real dataset: Indian Pines}

The AVIRIS Indian Pines dataset consists of $145 \times 145$ pixels from 200 spectral bands after removing the water absorption bands. There are sixteen classes of materials in the scene. For each of the 16 ground-truth classes, we randomly choose about $9 \%$ of labelled pixels as the dictionary, i.e. $\mathbf{D} \in$ $\mathbb{R}^{200 \times 957}$. The rest pixels are used for testing, i.e. $\mathbf{X}^{\text {test }} \in \mathbb{R}^{200 \times 9409}$. Similar experiment settings can also be found in [3 9, 16 18, 20] with different training/test samples and accordingly non-identical performance.

A summary of the numbers of training and test pixels for individual classes is given in Table 4. The false colour of the image averaging through all the bands, the 16 ground-truth classes, the training set and the test set are shown in Figures. 2(a) 2(d). 
Table 4: The Indian Pines dataset: Ground-truth label, class material, training set and test set. We use around ( $9 \%$ of all pixels) for training and the rest for testing.

\begin{tabular}{cr|rr}
\hline Class & Material & Training & Test \\
\hline 1 & Alfalfa & 5 & 49 \\
2 & Corn-notill & 132 & 1302 \\
3 & Corn-mintill & 77 & 757 \\
4 & Corn & 22 & 212 \\
5 & Grass-pasture & 46 & 451 \\
6 & Grass-trees & 69 & 678 \\
7 & Grass-pasture-mowed & 3 & 23 \\
8 & Hay-windrowed & 45 & 444 \\
9 & Oats & 2 & 18 \\
10 & Soybean-notill & 89 & 879 \\
11 & Soybean-mintill & 227 & 2241 \\
12 & Soybean-clean & 57 & 557 \\
13 & Wheat & 20 & 192 \\
14 & Woods & 119 & 1175 \\
15 & Buildings-grass-trees-drives & 35 & 345 \\
16 & Stone-steel-towers & 9 & 86 \\
\hline Total & & 957 & 9409 \\
\hline
\end{tabular}

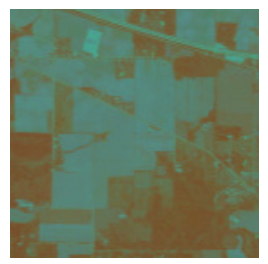

(a)

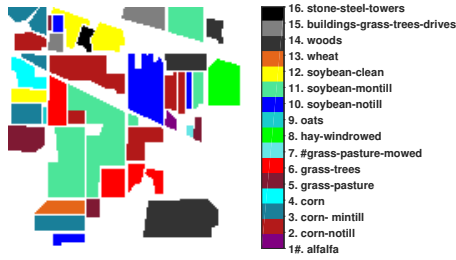

(b)

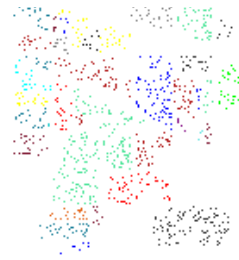

(c)

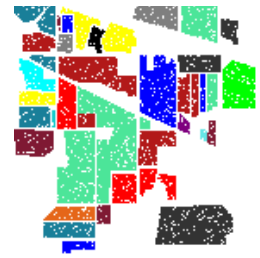

(d)

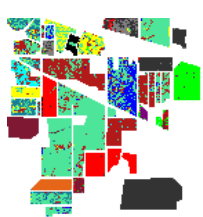

(e)

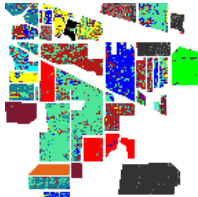

(f)

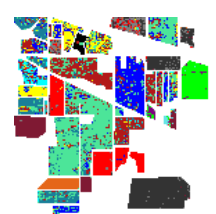

$(\mathrm{g})$

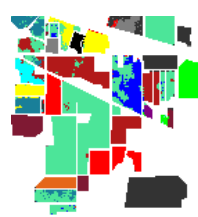

(h)

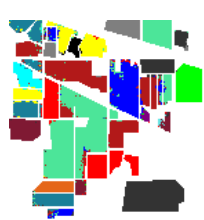

(i)

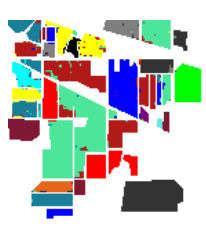

(j)

Figure 2: The Indian Pines dataset: (a) mean image shown in the false colour; (b) groundtruth labels; (c) training set (9\% pixels randomly chosen); (d) test set. Classification maps of (e) CM (NNLS), $O A=75.42$; (f) SM (OMP), $O A=74.79 ;(\mathrm{g}) \mathrm{CSM}(\mathrm{NN}-\mathrm{OMP}), O A$ $=74.83$; (h) JCM (FC-NNLS), $O A=84.88$; (i) JSM (SOMP), $O A=93.79$; (j) C-JSM $(\mathrm{NN}-\mathrm{SOMP}), O A=95.19$. 
For a more reliable evaluation, we perform the experiments by 10 times of random training/test splits. For illustration, the optimal parameters obtained by LOOCV of one random training/test split are listed in Table 5 . Note that the NNLS has no parameter to be tuned and hence no training process is required. For the OMP and NN-OMP, the tuned value of sparsity level $L$ is 5 ; for the FC-NNLS, the tuned value of window size $T$ is 25 $(5 \times 5)$; for the SOMP, the values of $L$ and $T$ are tuned to be 30 and 81 $(9 \times 9)$, respectively; and for the proposed NN-SOMP, the value of $L$ and $T$ are tuned to be 15 and $25(5 \times 5)$, respectively.

Table 5: Settings of parameters for the Indian Pines dataset in one random training/test split. The values of parameters are determined by LOOCV. "NA" stands for "not applicable".

\begin{tabular}{r|r|r|r|r|r|r}
\hline & NNLS & OMP & NN-OMP & FC-NNLS & SOMP & NN-SOMP \\
\hline$L$ & NA & 5 & 5 & NA & 30 & 15 \\
\hline$T$ & NA & NA & NA & 25 & 81 & 25 \\
\hline
\end{tabular}

\subsubsection{Classification performances}

The 10 overall classification $O A$ s of all six compared methods are recorded and box-plotted in Figure. 3 . For illustration purposes, we also randomly choose one of the 10 classification results and list the $O A, A A$ and $\kappa$ coefficient of all methods in Table 6, and depict the classification maps of the corresponding methods in Figure. $2(\mathrm{e}), 2(\mathrm{j})$, respectively.

From Figure. 3, we can observe two patterns. Firstly, we can observe that the proposed C-JSM (NN-SOMP) outperforms the other two joint models, JCM (FC-NNLS) and JSM (SOMP). Also, among the three single models, CSM (NN-OMP) performs the best, superior to CM (NNLS) and SM (OMP). These indicate that incorporating the non-negativity constraints into HSI classification can help to improve the performance of the 
sparse representation-based classifiers. Secondly, the proposed C-JSM (NNSOMP) performs the best among all the compared methods. It indicates that combining the non-negativity constraints and the joint sparse representation can improve the classification performance the most, compared with the representation with any single constraint, i.e. joint representation, sparse representation or non-negative representation.

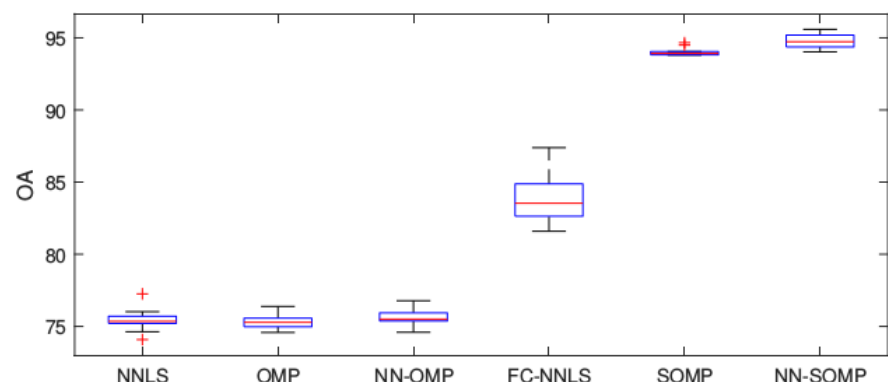

Figure 3: Boxplots of the overall classification accuracies (\%) of 3 single models (CM (NNLS), SM (OMP), CSM (NN-OMP)) and 3 joint models (JCM (FC-NNLS), JSM (SOMP), C-JSM (NN-SOMP)) on the Indian Pines dataset.

The one time classification results listed in Table 6 also show that the proposed C-JSM (NN-SOMP) outperforms other methods, which is aligned with our findings from the 10 times repeated random splits (Figure. 3). We also notice two special cases, with class 7 and class 9 , that the numbers of training samples are extremely small, i.e. 3 for class 7 and 2 for class 9 , as listed in Table 4. All methods except for C-JSM (NN-SOMP) do not perform very well on classifying these two tiny classes of HSI pixels. For the single models, i.e. CM (NNLS), SM (OMP) and CSM (NN-OMP), the bad performances may be due to the lack of training samples. For the joint models of JCM (FC-NNLS) and JSM (SOMP), the performances are even worse. Particularly in class 9, the classification accuracies of both models 
Table 6: The Indian Pines dataset: Ground-truth label and the classification accuracies (\%) obtained by CM (NNLS), SM (OMP), CSM (NN-OMP), JCM (FC-NNLS), JSM (SOMP) and C-JSM (NN-SOMP), respectively. The best performance is indicated in bold.

\begin{tabular}{r|r|r|r|r|r|r}
\hline Class & CM & SM & CSM & JCM & JSM & C-JSM \\
\hline 1 & 75.51 & 53.06 & 51.02 & 83.67 & 71.43 & $\mathbf{8 5 . 7 1}$ \\
2 & 72.66 & 62.98 & 62.83 & 83.26 & $\mathbf{9 4 . 2 4}$ & 93.86 \\
3 & 37.65 & 62.62 & 63.14 & 56.67 & 88.90 & $\mathbf{9 2 . 8 7}$ \\
4 & 48.11 & 40.57 & 41.51 & 79.25 & $\mathbf{9 2 . 4 5}$ & 88.21 \\
5 & 85.81 & 94.90 & 94.68 & 94.24 & 93.79 & $\mathbf{9 8 . 0 0}$ \\
6 & 96.31 & 93.36 & 93.22 & $\mathbf{9 9 . 7 1}$ & 98.97 & 98.38 \\
7 & 4.35 & 78.26 & 78.26 & 0 & 69.57 & $\mathbf{1 0 0}$ \\
8 & 98.20 & 95.05 & 95.05 & $\mathbf{1 0 0}$ & 99.77 & 99.77 \\
9 & 22.22 & 55.56 & 55.56 & 0 & 0 & $\mathbf{7 2 . 2 2}$ \\
10 & 36.63 & 72.47 & 73.49 & 42.78 & 80.55 & $\mathbf{9 5 . 4 5}$ \\
11 & 89.29 & 74.16 & 74.03 & $\mathbf{9 9 . 0 6}$ & 95.98 & 96.21 \\
12 & 61.04 & 54.76 & 54.04 & 84.56 & $\mathbf{9 1 . 3 8}$ & 88.51 \\
13 & 98.96 & 99.48 & 91.67 & $\mathbf{9 9 . 4 8}$ & $\mathbf{9 9 . 4 8}$ & 83.33 \\
14 & 98.98 & 92.68 & 92.34 & $\mathbf{9 9 . 7 4}$ & 98.89 & 97.70 \\
15 & 44.64 & 46.38 & 47.83 & 58.84 & $\mathbf{9 9 . 7 1}$ & 94.20 \\
16 & 87.21 & 88.37 & 88.37 & $\mathbf{1 0 0}$ & 96.51 & 89.53 \\
\hline \hline OA & 75.42 & 74.79 & 74.83 & 84.88 & 93.79 & $\mathbf{9 5 . 1 9}$ \\
$A A$ & 66.10 & 72.79 & 72.80 & 73.83 & 84.06 & $\mathbf{9 2 . 6 4}$ \\
$\kappa$ & 0.714 & 0.713 & 0.713 & 0.824 & 0.929 & $\mathbf{0 . 9 4 5}$ \\
\hline
\end{tabular}


are 0 . This is because class 7 and class 9 cover narrow regions in the Indian Pines HSIs (as shown in Figure. 2). The label of the central test pixel can be dominated by classes adjacent and thus misclassified. However, the proposed C-JSM (NN-SOMP) relives this spatial-over-smoothness caused by the local window strategy and outperforms the other five methods with substantial improvements: achieving 100\% against the second best $78.26 \%$ for class 7 and achieving $72.22 \%$ against the second best $55.56 \%$ for class 9 .

\subsubsection{Effects of parameters}

We further investigate the effects of tuning parameters on the performance of our proposed C-JSM (NN-SOMP). A sweep of the parameter space of sparsity level $L$ and window size $T$ is performed during the training phase. The sparse level $L$ is tuned from 5 to 80 and the window size $T$ ranges from 1 to $289(17 \times 17)$. The LOOCV result of C-JSM $($ NN-SOMP) is depicted in Figure. 4(a), Within the same parameter space ( $L$ and $T$ ), we also show the LOOCV result of JSM (SOMP) in Figure. 4(b) for comparison.

As shown in Figure. 4(a) and Figure. 4(b), we can easily see that the surface plot of $O A$ s for C-JSM (NN-SOMP) is much smoother than that of JSM (SOMP). It implies that C-JSM (NN-SOMP) is more stable than that of JSM (SOMP) in terms of the performance sensitivity to $L$ and $T$. More specifically, we split the 3-D view of the $O A$ surface of C-JSM (NN-SOMP) into two 2-D views, which are shown in Figure. 5(a) and Figure. 5(b). It can be observed that the window size $T$ dominates the performance of C-JSM whereas the effect of sparsity level $L$ on the classification performance is not as sensitive as $T$.

To further demonstrate the effect of sparsity level $L$, we perform classification on one of the 10 randomly split test dataset, by fixing the window 


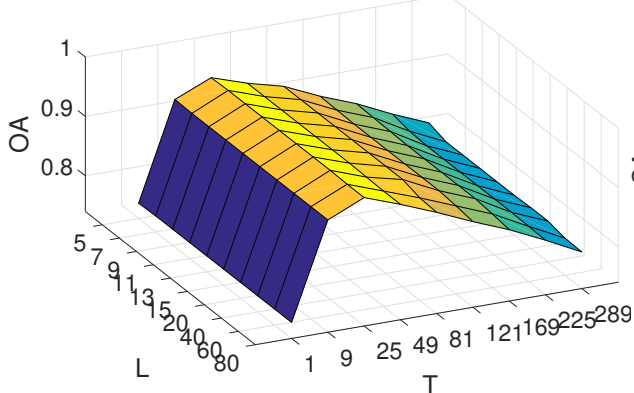

(a)

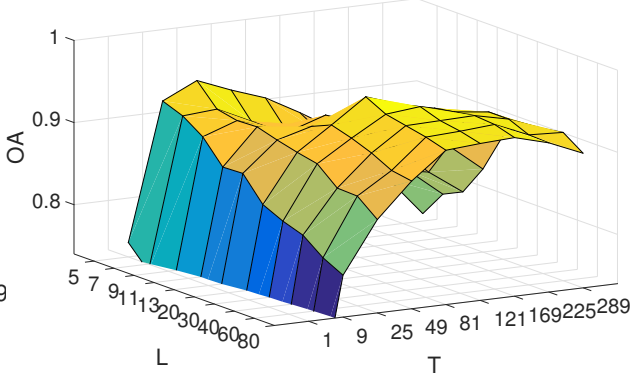

(b)

Figure 4: Overall classification accuracies over window size $T$ and sparsity level $L$ for (a) the proposed C-JSM (NN-SOMP) and (b) the JSM (SOMP) on the Indian Pines training dataset via LOOCV.
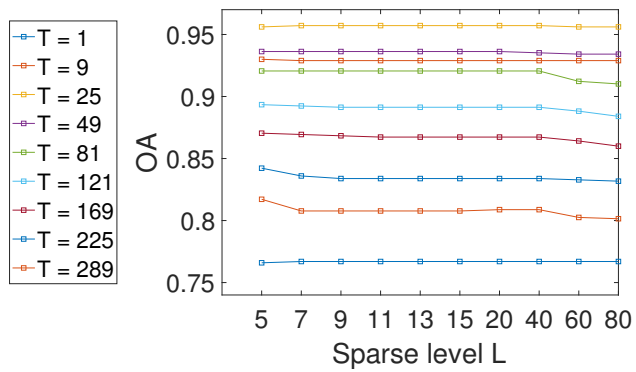

(a)

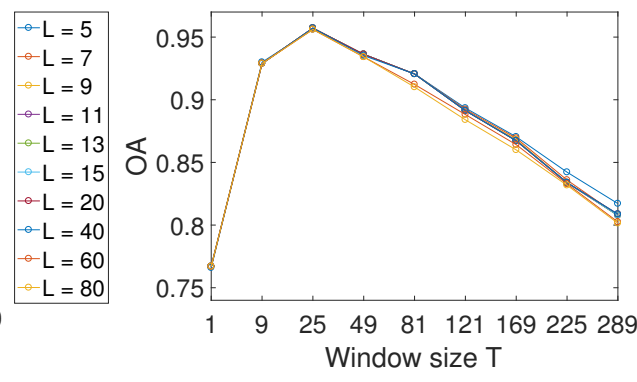

(b)

Figure 5: Effects of the sparsity $L$ and window size $T$ on the performance of the proposed C-JSM corresponding to Figure. 4(a). 
size $T$ to be $25(5 \times 5)$ as tuned by LOOCV. This test dataset is the same as the one used in Table 6 and Figure. 2. We set the level of sparsity $L$ from 5 to 80 and depict the obtained $O A$ s in Figure. 6(a). Accordingly, we record the real sparsity $L^{\prime}$ obtained in different settings of $L$. Since different test HSI pixels have different real sparsities $L^{\prime}$ under a defined $L$, we record and box-plot them in Figure. 6(b).

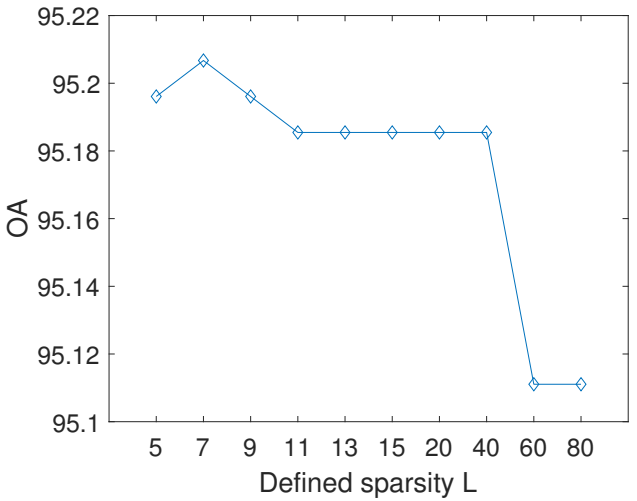

(a)

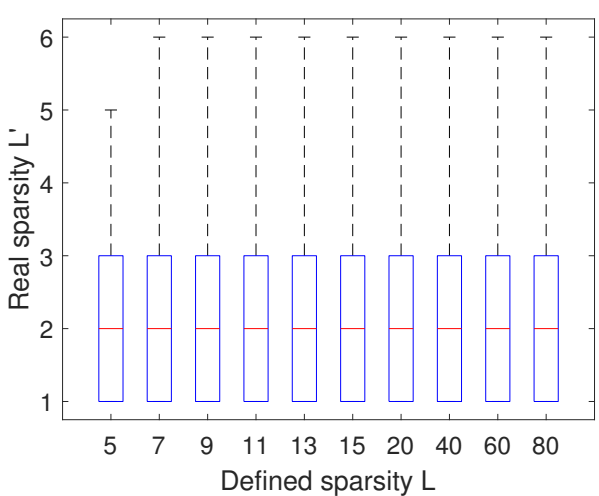

(b)

Figure 6: Window size $T=5$ on the test dataset of Indian Pines: (a) classification performance (overall accuracies) with sparsity level $L$; (b) the real sparsity level $L^{\prime}$ obtained from the test results with sparsity level $L$.

It can be seen that, although the best $O A$ occurs at $L=7$ when $T$ is fixed to be 25 , the performance only changes slightly with the defined sparsity $L$, where the $O A$ changes only from $95.11 \%$ to $95.21 \%$. Therefore the $O A=95.19 \%$ of C-JSM (NN-SOMP) listed in Table 6 with $L=5$ is in the range of the stable performance, where the parameters are tuned by LOOCV and the testing results are reliable.

On the other hand, we can observe that the obtained sparsity $L^{\prime}$ ranges from 1 to 6 , and the median value of them is around 2 no matter what values 
the defined sparsity $L$ are. Furthermore, the obtained maximal sparsity $L^{\prime}$ converges to 6 when the defined sparsity $L$ is over 6 , as shown in Figure. 6(b), This explains why the performance of C-JSM (NN-SOMP) is not so sensitive to the setting of sparsity $L$. However, the setting of sparsity $L$ still gives some room for each test HSI pixel to adaptively choose their optimal sparsity level and hence can achieve a stable and reliable classification performance.

\subsubsection{Sparseness and non-negativity}

We next demonstrate the effects of sparsity and non-negativity on all the compared methods by adopting a similar presentation in [50] and depicting the results in Figures. 77[10. The classification results of all methods are obtained in parameter settings listed in Table 5. For comparative purposes, we randomly select two test HSI pixels which belong to class 10 and are located in $(48,31)$ and $(53,88)$ : one is correctly classified by all six methods and the other is only correctly classified by C-JSM (NN-SOMP).

For pixel $(48,31)$, the associated class-wise residuals obtained by all six methods are shown in Figure. 7. We can observe that the pixel is correctly classified by all six methods into class 10 , which has the minimum residuals and is indeed the ground-truth class. Among the six methods, CSM in Figure. 7(c) and C-JSM in Figure. 7(f), both of which contains both sparse and non-negative constraints, perform the best with the true class (with the smallest residual) and the most stable relative to other classes (with all large residuals).

To investigate further, we plot the obtained coefficients of this pixel in Figure. 8, Because for the single models (CM, SM and CSM) there is only one coefficient vector for the test HSI pixel $\mathbf{x}$, there is only one colour shown in the plots of coefficients; whereas for the joint models (JCM, JSM and 


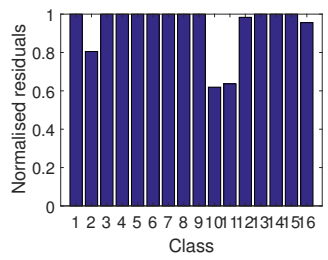

(a)

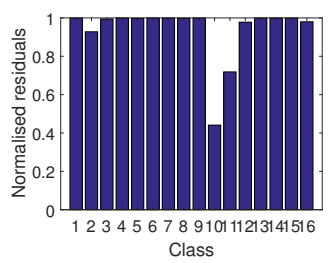

(d)

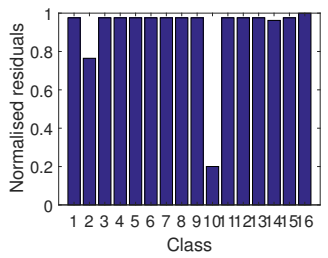

(b)

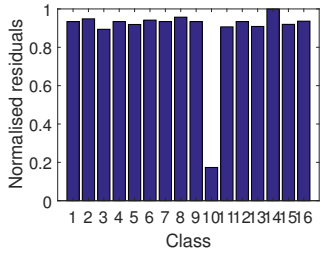

(e)

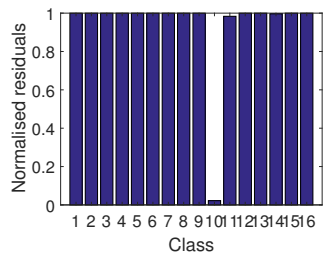

(c)

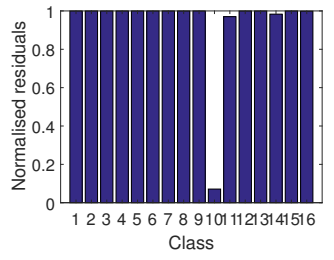

(f)

Figure 7: Normalised residuals for each class for the pixel located at $(48,31)$ by (a) CM, (b) SM, (c) CSM, (d) JCM, (e) JSM and (f) C-JSM. The ground-truth label is class 10. The test pixel is correctly identified by all six methods.

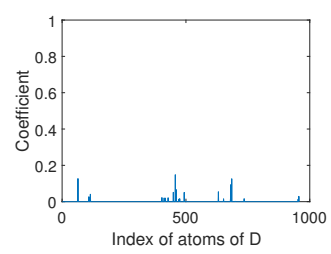

(a)

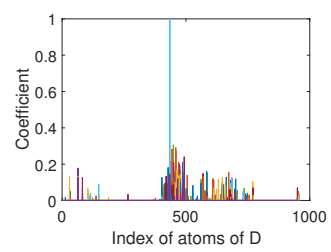

(d)

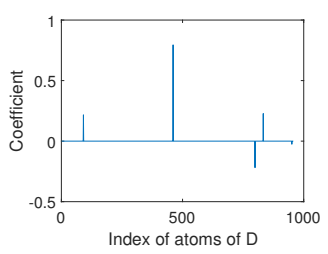

(b)

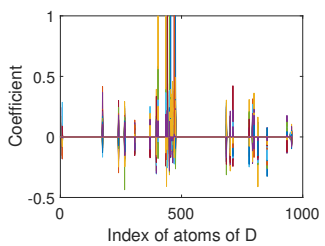

(e)

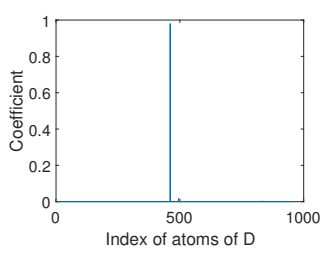

(c)

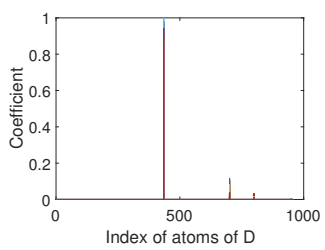

(f)

Figure 8: Estimated coefficients for the pixel located at $(48,31)$ by (a) CM, (b) SM, (c) CSM, (d) JCM, (e) JSM and (f) C-JSM. The ground-truth label is class 10. The test pixel is correctly identified by all six methods. 
C-JSM), the label of the central test pixel $\mathbf{x}_{c}$ is jointly determined by its local window $\mathbf{X}$, hence we plot all the coefficient vectors of the pixels in the window in different colours. In addition, since the test HSI pixel actually belongs to class 10 , we expect to see that the coefficients mainly lie within the sub-dictionary of class 10, where the atom indices range from 402 to 490.

From Figure. 8, we can observe that, although all methods can identify the correct class 10 for pixel $(48,31)$, the coefficient vectors obtained by different methods are remarkably different, and again, the most neat (sparse) performances are with C-JSM (Figure. 8(f)] and CSM (Figure. 8(c)]. This also indicates that incorporating the non-negativity constraint into the sparse model is beneficial, which can produce a more sparse representation.

However, the sparse and non-negative constraints are not the only two factors that may ensure correct label identification for HSIs. As illustrated in Figure. 9 and Figure. 10, for a test HSI pixel located in $(53,88)$, only the proposed C-JSM identifies its label as class 10 correctly (Figure. 9(f)]. In C-JSM, the non-zero elements of the coefficients vectors of all pixels within the neighbourhood window mainly lie in class 10 and the label of the central test pixel is jointly determined by the minimal residuals, which belongs to class 10 (Figure. 10(f), In contrast, although the coefficient vector obtained by CSM in Figure. 10(c) is non-negative and most sparse, it lies in class 11, a wrong class (Figure. 9(c)]. This illustrates that the joint representation of neighbouring pixels on top of the sparsity and non-negativity can positively contribute to the classification performance for HSIs, and hence the proposed C-JSM outperforms others. 


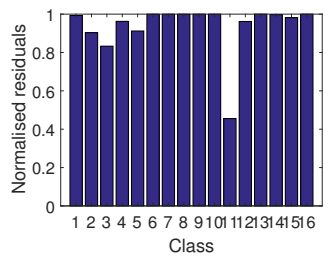

(a)

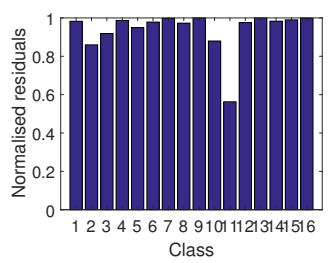

(d)

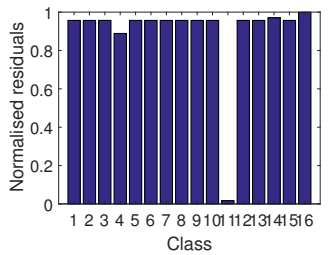

(b)

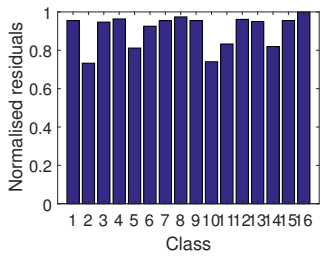

(e)

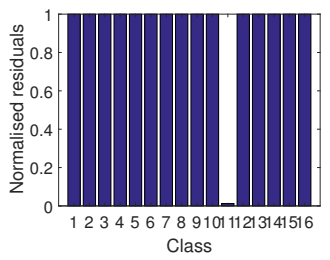

(c)

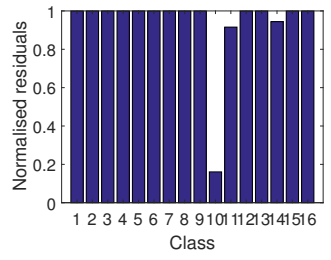

(f)

Figure 9: Normalised residuals for each class for the pixel located at $(53,88)$ by (a) CM, (b) SM, (c) CSM, (d) JCM, (e) JSM and (f) C-JSM. The ground-truth label is class 10. The test pixel is only correctly identified by our proposed C-JSM.

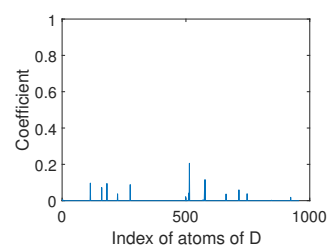

(a)

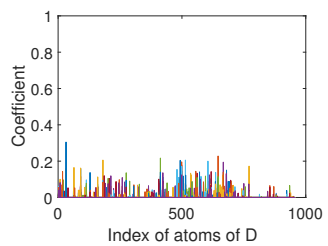

(d)

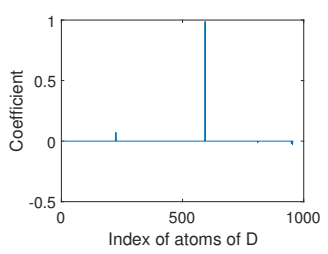

(b)

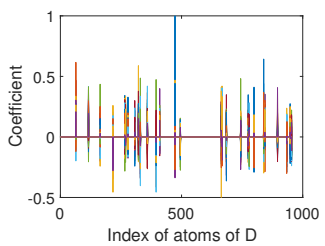

(e)

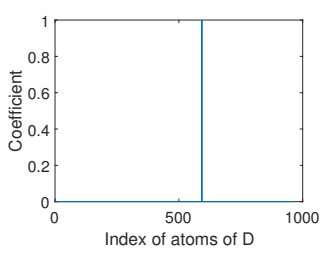

(c)

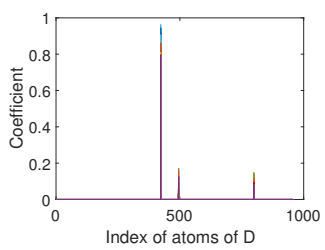

(f)

Figure 10: Estimated coefficients for the pixel located at $(53,88)$ by (a) CM, (b) SM, (c) CSM, (d) JCM, (e) JSM and (f) C-JSM. The ground-truth label is class 10. The test pixel is only correctly identified by our proposed C-JSM. 


\subsection{Real dataset: University of Pavia}

The ROSIS University of Pavia dataset consists of $610 \times 340$ pixels from 103 spectral bands, with nine ground-truth labels. We randomly choose only $1 \%$ of labelled samples from each class for constructing the dictionary, i.e. $\mathbf{D} \in \mathbb{R}^{103 \times 432}$, and use the rest HSI pixels for testing, i.e. $\mathbf{X}^{\text {test }} \in$ $\mathbb{R}^{103 \times 42344}$. A summary of this dataset is given in Table 7. Again, a false colour image averaging across all spectral bands, the nine ground-truth classes, the training set and the test set are shown in Figures. $11(\mathrm{a}), 11(\mathrm{~d})$,

Table 7: The Pavia University dataset: Ground-truth labels, class material, the training set and the test set.

\begin{tabular}{cr|rr}
\hline Class & materials & Training & Test \\
\hline 1 & Asphalt & 67 & 6564 \\
2 & Meadows & 187 & 18462 \\
3 & Gravel & 21 & 2078 \\
4 & Trees & 31 & 3033 \\
5 & Painted metal sheets & 14 & 1331 \\
6 & Bare soil & 51 & 4978 \\
7 & Bitumen & 14 & 1316 \\
8 & Self-blocking bricks & 37 & 3645 \\
9 & Shadows & 10 & 937 \\
\hline Total & & 432 & 42344 \\
\hline
\end{tabular}

For a reliable evaluation, the experiments are also performed by 10 times random train/test splits, as with the Indian Pines dataset in section 6.4. For illustration, the optimal values of parameters tuned by LOOCV using one time train/test random spilt are listed in Table 8. The $O A$ s of all six compared methods are box-plotted in Figure. 12, we also randomly select one of 10 classification results and illustrate them in Table 9 and Figure. $11(\mathrm{e})$ $11(\mathrm{j})$

Once again, we can observe that the proposed C-JSM (NN-SOMP) outperforms other methods. We also note that in Figure. 12 the performance of CM (NNLS) is better than that of SM (OMP) and of CSM (NN-OMP), 


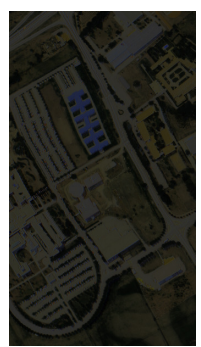

(a)

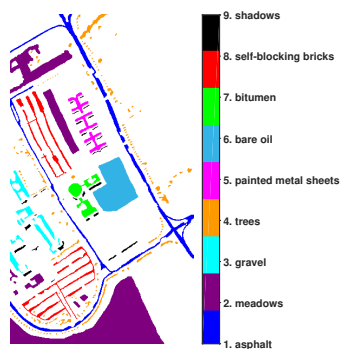

(b)

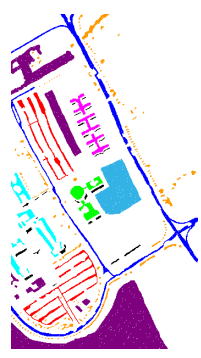

(d)

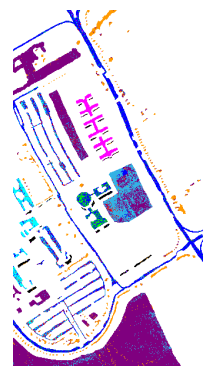

(e)

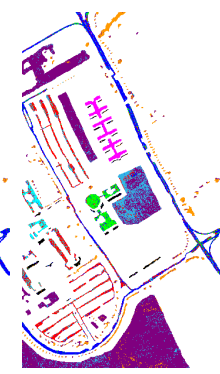

(f)

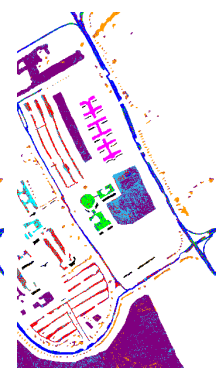

(g)

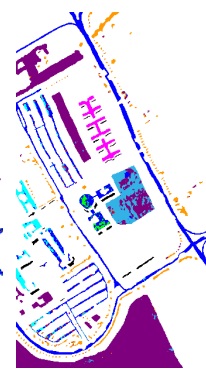

(h) (c)

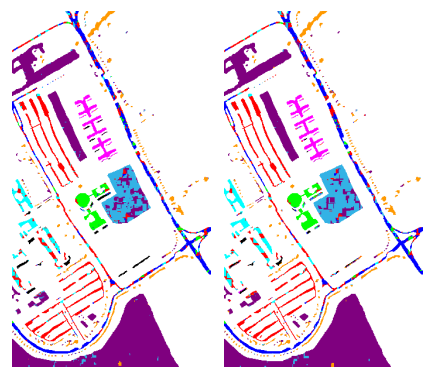

(i)

(j)

Figure 11: The University of Pavia dataset: (a) mean image shown in the false colour; (b) ground-truth labels; (c) training set (1\% pixels randomly chosen); (d) test set. Classification maps of (e) CM (NNLS), $O A=78.65$; (f) $\mathrm{SM}(\mathrm{OMP}), O A=78.72$; (g) CSM $(\mathrm{NN}-\mathrm{OMP}), O A=78.75$; (h) JCM (FC-NNLS), $O A=82.81$; (i) JSM (SOMP), $O A=$ 84.91; (j) C-JSM (NN-SOMP), $O A=86.53$.

Table 8: Settings of parameters for the University of Pavia dataset in one random training/test split. The values parameters are determined by LOOCV. "NA" stands for "not applicable".

\begin{tabular}{r|r|r|r|r|r|r}
\hline & NNLS & OMP & NN-OMP & FC-NNLS & SOMP & NN-SOMP \\
\hline$L$ & NA & 5 & 5 & NA & 10 & 3 \\
\hline$T$ & NA & NA & NA & 9 & 49 & 81 \\
\hline
\end{tabular}




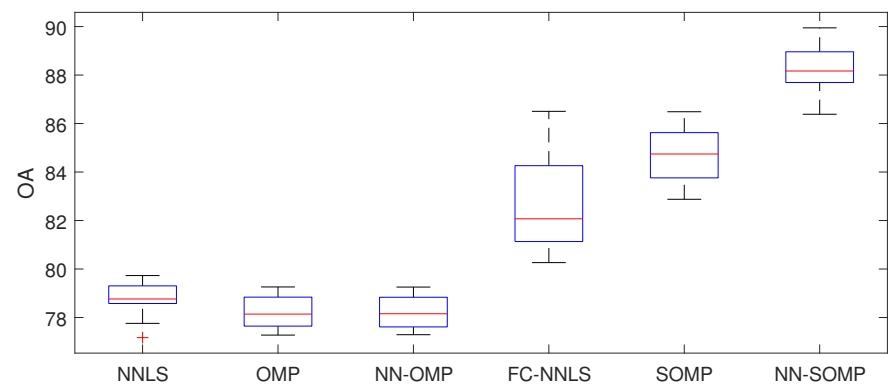

Figure 12: Boxplots of the overall classification accuracies (\%) of CM (NNLS), SM (OMP), CSM (NN-OMP), JCM (FC-NNLS), JSM (SOMP) and C-JSM (NN-SOMP) on the University of Pavia dataset.

Table 9: The University of Pavia dataset: Ground-truth label and the classification accuracies (\%) obtained by CM (NNLS), SM (OMP), CSM (NN-OMP), JCM (FC-NNLS), JSM (SOMP) and C-JSM (NN-SOMP), respectively. The best performance is indicated in bold.

\begin{tabular}{r|r|r|r|r|r|r}
\hline Class & CM & SM & CSM & JCM & JSM & C-JSM \\
\hline 1 & 85.65 & 70.75 & 70.81 & $\mathbf{9 8 . 9 2}$ & 57.46 & 59.83 \\
2 & 93.97 & 92.82 & 92.82 & $\mathbf{9 9 . 4 0}$ & 98.14 & 98.55 \\
3 & 62.70 & 45.62 & 45.62 & 69.30 & 70.12 & $\mathbf{7 7 . 4 8}$ \\
4 & 87.97 & 77.28 & 77.05 & $\mathbf{9 3 . 1 4}$ & 80.25 & 83.65 \\
5 & 99.77 & 99.25 & 99.25 & $\mathbf{1 0 0 . 0 0}$ & $\mathbf{1 0 0 . 0 0}$ & $\mathbf{1 0 0 . 0 0}$ \\
6 & 58.00 & 47.91 & 47.89 & 62.82 & 70.65 & $\mathbf{8 0 . 6 3}$ \\
7 & 42.33 & 77.43 & 77.43 & 28.12 & 92.63 & $\mathbf{9 5 . 7 4}$ \\
8 & 21.10 & 74.29 & 74.29 & 7.05 & 93.94 & $\mathbf{9 5 . 3 4}$ \\
9 & 87.41 & 88.26 & 89.97 & 92.74 & 72.89 & 31.06 \\
\hline$O A$ & 78.65 & 78.72 & 78.75 & 82.81 & 84.91 & $\mathbf{8 6 . 5 3}$ \\
$A A$ & 70.99 & 74.85 & 75.01 & 72.39 & $\mathbf{8 1 . 7 9}$ & 80.25 \\
$\kappa$ & 0.712 & 0.714 & 0.714 & 0.765 & 0.798 & $\mathbf{0 . 8 2 0}$ \\
\hline
\end{tabular}


a pattern different from the results shown for the Indian Pines dataset. As we have analysed in section 6.4.3, sparse and non-negative representations only may still be insufficient to produce a stable and correct classification. On the other hand, C-JSM incorporates the sparse and non-negativity constraints into the joint modelling of neighbouring pixels, and hence is capable of providing a more sparse representation and a more stable classification performance.

\subsection{Running time comparison}

Table 10: Running time (sec/pixel) spent on testing the Indian Pines dataset, settings of which are shown in Table 4 and Table 5 for 9409 test pixels.

\begin{tabular}{r|r|r|r|r|r|r}
\hline & NNLS & OMP & NN-OMP & FC-NNLS & SOMP & NN-SOMP \\
\hline Time & 0.0058 & 0.0175 & 0.0017 & 0.1195 & 0.0737 & 0.0392 \\
\hline
\end{tabular}

We present the time costs for executing the compared algorithms. All experiments are performed by a Intel i7-3370 CPU using single thread on the platform of MATLAB R2016b. Table 10 shows the running time of each method for the Indian Pines dataset. The time is recorded as second per HSI pixel.

First, we can observe that, among the single models, NN-OMP takes less time than NNLS and OMP. In fact the obtained coefficients of NNOMP are more sparse than the others, as indicated by Figure. $8(\mathrm{a}), 8(\mathrm{c})$ and Figure. $10(\mathrm{a})$ 10(c), It implies that the computational burden is lessened by NN-OMP. Secondly, among the joint models, our proposed NN-SOMP is more time-efficient than FC-NNLS and SOMP. It is also because the obtained coefficients from NN-SOMP are more sparse than the others, as indicated by Figure. $8(\mathrm{~d}), 8(\mathrm{f})$ and Figure. $10(\mathrm{~d}), 10(\mathrm{f})$, and hence the computational costs are reduced. 


\subsection{Further remarks}

It is worth noting that several literatures have studied the relationship between the sparsity and non-negativity [42, 51]. It has been shown that the non-negative least squares (NNLS) may be able to produce sufficient sparse recovery, without further imposing the sparse regularisations. However, we remark that this does not imply that the performances of NNLS and the sparsely regularised NNLS are the same, particular for the classification problems that are the focus of this paper. That is, the distinct classification performances of the compared methods of different constraints in this paper do not conflict the existing findings in [42, 51].

\section{Conclusion and future work}

To sum up, by considering the non-negativity of coefficients for the jointly sparse representation of HSI pixels, a new model called cone-based joint sparse model (J-CSM) has been proposed in this paper. To solve the C-JSM, a new algorithm, called non-negative simultaneous orthogonal matching pursuit (NN-SOMP), has also been proposed. The C-JSM incorporates the non-negativity of coefficients, as well as the spatial coherence of the HSI pixels, into one model, yielding a more sparse and stable representation for the test HSI pixel whose label is jointly determined by its neighbouring pixels. As a result, the classification performance of the JSM is enhanced by the proposed C-JSM.

We notice that the proposed C-JSM may not completely solve the problems that are caused by the local window scheme. Specifically, the square shape of the window adopted in this paper indeed introduces bias into the

joint models and may cause spatial-over-smoothness. That is, the classifica- 
tion of the HSI pixel may not have a promising edge-preserving performance. As aforementioned in the introduction (section 1), several literatures have studied the improvement of the JSM by adopting size/shape adaptive windows [6 9]. The proposed C-JSM can also be collaboratively conducted with the window adaptation strategies for enhancing the classification performance. On the other hand, it is also desired to exploit the non-linearity representation, such as kernelisation [4, 5], of the HSIs together with the non-negativity constraints for the joint sparse models. These two directions are our future research on the proposed C-JSM.

\section{Acknowledgment}

This work was partially supported by University College London's Security Science Doctoral Training Centre under Engineering and Physical Sciences Research Council (EPSRC) grant EP/G037264/1.

\section{References}

[1] J. Wright, Y. Ma, J. Mairal, G. Sapiro, T. S. Huang, S. Yan, Sparse representation for computer vision and pattern recognition, Proceedings of the IEEE 98 (6) (2010) 1031-1044.

[2] Y. Han, Y. Yang, Y. Yan, Z. Ma, N. Sebe, X. Zhou, Semisupervised feature selection via spline regression for video semantic recognition, IEEE Transactions on Neural Networks and Learning Systems 26 (2) (2015) 252-264.

[3] Y. Chen, N. M. Nasrabadi, T. D. Tran, Hyperspectral image classification using dictionary-based sparse representation, IEEE Transactions on Geoscience and Remote Sensing 49 (10) (2011) 3973-3985. 
[4] Y. Chen, N. M. Nasrabadi, T. D. Tran, Hyperspectral image classification via kernel sparse representation, IEEE Transactions on Geoscience and Remote sensing 51 (1) (2013) 217-231.

[5] J. Liu, Z. Wu, Z. Wei, L. Xiao, L. Sun, Spatial-spectral kernel sparse representation for hyperspectral image classification, IEEE Journal of Selected Topics in Applied Earth Observations and Remote Sensing 6 (6) (2013) 2462-2471.

[6] H. Zhang, J. Li, Y. Huang, L. Zhang, A nonlocal weighted joint sparse representation classification method for hyperspectral imagery, IEEE Journal of Selected Topics in Applied Earth Observations and Remote Sensing 7 (6) (2014) 2056-2065.

[7] Y. Y. Tang, H. Yuan, L. Li, Manifold-based sparse representation for hyperspectral image classification, IEEE Transactions on Geoscience and Remote Sensing 52 (12) (2014) 7606-7618.

[8] L. Fang, S. Li, X. Kang, J. A. Benediktsson, Spectral-spatial classification of hyperspectral images with a superpixel-based discriminative sparse model, IEEE Transactions on Geoscience and Remote Sensing 53 (8) (2015) 4186-4201.

[9] J. Li, H. Zhang, L. Zhang, Efficient superpixel-level multitask joint sparse representation for hyperspectral image classification, IEEE Transactions on Geoscience and Remote Sensing 53 (10) (2015) 53385351.

[10] Y. Zhang, B. Du, L. Zhang, A sparse representation-based binary hypothesis model for target detection in hyperspectral images, IEEE 
Transactions on Geoscience and Remote Sensing 53 (3) (2015) 13461354.

[11] X. Wang, Y. Gao, Y. Cheng, A non-negative sparse semi-supervised dimensionality reduction algorithm for hyperspectral data, Neurocomputing 188 (2016) 275-283.

[12] H. Wang, T. Celik, Sparse representation-based hyperspectral data processing: Lossy compression, IEEE Journal of Selected Topics in Applied Earth Observations and Remote Sensing 10 (5) (2017) 2036-2045.

[13] Y. Zhang, B. Du, L. Zhang, T. Liu, Joint sparse representation and multitask learning for hyperspectral target detection, IEEE Transactions on Geoscience and Remote Sensing 55 (2) (2017) 894-906.

[14] H. Li, C. Li, C. Zhang, Z. Liu, C. Liu, Hyperspectral image classification with spatial filtering and $l_{(2,1)}$ norm, Sensors 17 (2) (2017) 314.

[15] J. A. Tropp, A. C. Gilbert, Signal recovery from random measurements via orthogonal matching pursuit, IEEE Transactions on Information Theory 53 (12) (2007) 4655-4666.

[16] A. Soltani-Farani, H. Rabiee, S. Hosseini, Spatial-aware dictionary learning for hyperspectral image classification, IEEE Transactions on Geoscience and Remote Sensing 53 (1) (2015) 527-541.

[17] Z. Wang, N. M. Nasrabadi, T. S. Huang, Spatial-spectral classification of hyperspectral images using discriminative dictionary designed by learning vector quantization, IEEE Transactions on Geoscience and Remote Sensing 52 (8) (2014) 4808-4822. 
[18] X. Sun, N. M. Nasrabadi, T. D. Tran, Task-driven dictionary learning for hyperspectral image classification with structured sparsity constraints, IEEE Transactions on Geoscience and Remote Sensing 53 (8) (2015) 4457-4471.

[19] Z. Wang, N. M. Nasrabadi, T. S. Huang, Semisupervised hyperspectral classification using task-driven dictionary learning with Laplacian regularization, IEEE Transactions on Geoscience and Remote Sensing 53 (3) (2015) 1161-1173.

[20] Z. Wang, J. Liu, J.-H. Xue, Joint sparse model-based discriminative K-SVD for hyperspectral image classification, Signal Processing 133 (2017) 144-155.

[21] J. A. Tropp, A. C. Gilbert, M. J. Strauss, Algorithms for simultaneous sparse approximation. part i: Greedy pursuit, Signal Processing 86 (3) (2006) 572-588.

[22] V. P. Pauca, J. Piper, R. J. Plemmons, Nonnegative matrix factorization for spectral data analysis, Linear Algebra and Its Applications 416 (1) (2006) 29-47.

[23] L. Miao, H. Qi, Endmember extraction from highly mixed data using minimum volume constrained nonnegative matrix factorization, IEEE Transactions on Geoscience and Remote Sensing 45 (3) (2007) 765-777.

[24] X. Liu, W. Xia, B. Wang, L. Zhang, An approach based on constrained nonnegative matrix factorization to unmix hyperspectral data, IEEE Transactions on Geoscience and Remote Sensing 49 (2) (2011) 757-772. 
[25] Z. Yang, G. Zhou, S. Xie, S. Ding, J.-M. Yang, J. Zhang, Blind spectral unmixing based on sparse nonnegative matrix factorization, IEEE Transactions on Image Processing 20 (4) (2011) 1112-1125.

[26] J. Liu, J. Zhang, Y. Gao, C. Zhang, Z. Li, Enhancing spectral unmixing by local neighborhood weights, IEEE Journal of Selected Topics in Applied Earth Observations and Remote Sensing 5 (5) (2012) 1545-1552.

[27] N. Wang, B. Du, L. Zhang, An endmember dissimilarity constrained non-negative matrix factorization method for hyperspectral unmixing, IEEE Journal of Selected Topics in Applied Earth Observations and Remote Sensing 6 (2) (2013) 554-569.

[28] X. Lu, H. Wu, Y. Yuan, P. Yan, X. Li, Manifold regularized sparse NMF for hyperspectral unmixing, IEEE Transactions on Geoscience and Remote Sensing 51 (5) (2013) 2815-2826.

[29] X. Lu, H. Wu, Y. Yuan, Double constrained NMF for hyperspectral unmixing, IEEE Transactions on Geoscience and Remote Sensing 52 (5) (2014) 2746-2758.

[30] C. Févotte, N. Dobigeon, Nonlinear hyperspectral unmixing with robust nonnegative matrix factorization, IEEE Transactions on Image Processing 24 (12) (2015) 4810-4819.

[31] Y. Yuan, M. Fu, X. Lu, Substance dependence constrained sparse NMF for hyperspectral unmixing, IEEE Transactions on Geoscience and Remote Sensing 53 (6) (2015) 2975-2986.

[32] Y. Yuan, Y. Feng, X. Lu, Projection-based NMF for hyperspectral un- 
mixing, IEEE Journal of Selected Topics in Applied Earth Observations and Remote Sensing 8 (6) (2015) 2632-2643.

[33] R. Liu, B. Du, L. Zhang, Hyperspectral unmixing via double abundance characteristics constraints based NMF, Remote Sensing 8 (6) (2016) 464.

[34] B. Du, S. Wang, N. Wang, L. Zhang, D. Tao, L. Zhang, Hyperspectral signal unmixing based on constrained non-negative matrix factorization approach, Neurocomputing 204 (2016) 153-161.

[35] W. He, H. Zhang, L. Zhang, Total variation regularized reweighted sparse nonnegative matrix factorization for hyperspectral unmixing, IEEE Transactions on Geoscience and Remote Sensing 55 (7) (2017) 3909-3921.

[36] W. He, H. Zhang, L. Zhang, Sparsity-regularized robust non-negative matrix factorization for hyperspectral unmixing, IEEE Journal of Selected Topics in Applied Earth Observations and Remote Sensing 9 (9) (2016) 4267-4279.

[37] W. Dong, F. Fu, G. Shi, X. Cao, J. Wu, G. Li, X. Li, Hyperspectral image super-resolution via non-negative structured sparse representation, IEEE Transactions on Image Processing 25 (5) (2016) 2337-2352.

[38] Z. Wang, R. Zhu, K. Fukui, J.-H. Xue, Matched shrunken cone detector (MSCD): Bayesian derivations and case studies for hyperspectral target detection, IEEE Transactions on Image Processing 26 (11) (2017) 54475461. 
[39] Z. Wang, Essays on hyperspectral image analysis: classification and target detection, Ph.D. thesis, University College London (UCL) (2017). URL http://discovery.ucl.ac.uk/id/eprint/1547605

[40] C. Lawson, R. Hanson, Solving Least Squares Problems, Society for Industrial and Applied Mathematics, 1995.

URL http://epubs .siam .org/doi/abs/10.1137/1.9781611971217

[41] R. Bro, S. De Jong, A fast non-negativity-constrained least squares algorithm, Journal of Chemometrics 11 (5) (1997) 393-401.

[42] A. M. Bruckstein, M. Elad, M. Zibulevsky, On the uniqueness of nonnegative sparse solutions to underdetermined systems of equations, IEEE Transactions on Information Theory 54 (11) (2008) 4813-4820.

[43] M. Yaghoobi, D. Wu, M. E. Davies, Fast non-negative orthogonal matching pursuit, IEEE Signal Processing Letters 22 (9) (2015) 12291233.

[44] Q. Shi, B. Du, L. Zhang, Spatial coherence-based batch-mode active learning for remote sensing image classification, IEEE Transactions on Image Processing 24 (7) (2015) 2037-2050.

[45] M. H. Van Benthem, M. R. Keenan, Fast algorithm for the solution of large-scale non-negativity-constrained least squares problems, Journal of Chemometrics 18 (10) (2004) 441-450.

[46] D. Leviatan, V. N. Temlyakov, Simultaneous approximation by greedy algorithms, Advances in Computational Mathematics 25 (1-3) (2006) $73-90$. 
[47] S. F. Cotter, B. D. Rao, K. Engan, K. Kreutz-Delgado, Sparse solutions to linear inverse problems with multiple measurement vectors, IEEE Transactions on Signal Processing 53 (7) (2005) 2477-2488.

[48] P. R. Foundation, A freeware multispectral image data analysis system, https://engineering.purdue.edu/ biehl/MultiSpec/ hyperspectral.html, [Online; accessed 22-July-2014] (2014).

[49] J. A. Richards, X. Jia, Remote Sensing Digital Image Analysis: An Introduction, New York: Springer-Verlag, 2006.

[50] J. Li, H. Zhang, Y. Huang, L. Zhang, Hyperspectral image classification by nonlocal joint collaborative representation with a locally adaptive dictionary, IEEE Transactions on Geoscience and Remote Sensing 52 (6) (2014) 3707-3719.

[51] M. Slawski, M. Hein, Non-negative least squares for high-dimensional linear models: Consistency and sparse recovery without regularization, Electronic Journal of Statistics 7 (2013) 3004-3056. 